\title{
Exposure Start Date
}

National Cancer Institute

\section{Source}

National Cancer Institute. Exposure Start Date. NCI Thesaurus. Code C83127.

The date on which the exposure begins. 